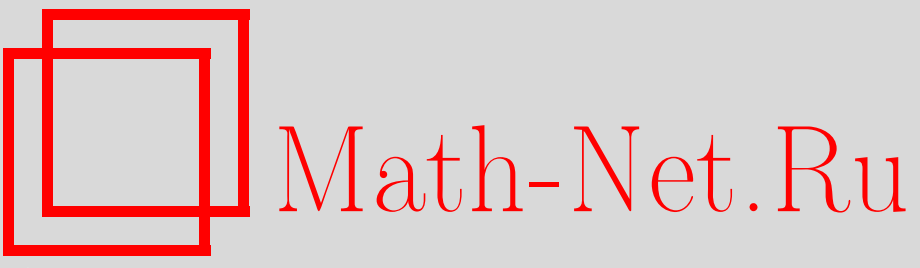

A. Ya. Narmanov, A. N. Zoyidov, On the group of diffeomorphisms of foliated manifolds, Vestn. Udmurtsk. Univ. Mat. Mekh. Komp. Nauki, 2020, Volume 30, Issue $1,49-58$

DOI: https://doi.org/10.35634/vm200104

Use of the all-Russian mathematical portal Math-Net.Ru implies that you have read and agreed to these terms of use

http: //www. mathnet.ru/eng/agreement

Download details:

IP : 3.95 .254 .165

April 26, 2023, 13:37:35 
MSC2010: 22A05, 54H15, 57R50

\author{
(C) A. Y. Narmanov, A. N. Zoyidov
}

\title{
ON THE GROUP OF DIFFEOMORPHISMS OF FOLIATED MANIFOLDS
}

Now the foliations theory is intensively developing branch of modern differential geometry, there are numerous researches on the foliation theory. The purpose of our paper is study the structure of the group Dif $f_{F}(M)$ of diffeomorphisms and the group $\operatorname{Iso}_{F}(M)$ of isometries of foliated manifold $(M, F)$. It is shown the group $\operatorname{Diff}_{F}(M)$ is closed subgroup of the group Diff $(M)$ of diffeomorphisms of the manifold $M$ in compact-open topology and also it is proven the group $I \operatorname{so}_{F}(M)$ is Lie group. It is introduced new topology on $\operatorname{Diff}_{F}(M)$ which depends on foliation $F$ and called $F$ - compact open topology. It's proven that some subgroups of the group $\operatorname{Dif} f_{F}(M)$ are topological groups with $F$-compact open topology.

Keywords: manifold, foliation, group of diffeomorphisms, compact open topology.

DOI: $10.35634 / \mathrm{vm} 200104$

\section{Introduction}

The set $\operatorname{Diff}(M)$ of all diffeomorphisms of manifold $M$ onto itself is the group related to composition and inverse mapping. The diffeomorphism group of smooth manifolds are of great importance in differential geometry and in analysis. The fundamental works in this area are the studies of V. I. Arnold, A. M. Lukatsky, H. Omori [1, 6, 7, 15, 16]. Intensive development of the theory of groups of diffeomorphisms began after the work of V. I. Arnold, in which it was shown that the motions of an ideal incompressible fluid are geodesic on a group of diffeomorphisms that preserve volume element.

When $M$ is a finite dimensional manifold in 1939 was proven that the group of isometries $I(M)$ of riemannian manifold $M$ is Lie group [9].

H. Omori defined the differential structure on group of diffeomorphisms compact manifold which is weaker than that structure of Lie group in the classical sense. This differentiable structure is called ILH (inverse limit of Hilbert) Lie group. H. Omori showed that ILH Lie group of diffeomorphisms acting on compact manifold transitively and primitively can only be the following: the whole group of diffeomorphisms, the group of diffeomorphisms preserving the volume element, the group of symplectic diffeomorphisms, the group of contact diffeomorphisms. The questions of finite generation of diffeomorphism groups and curvature properties of ILH - Lie groups of diffeomorphisms are investigated by A. M. Lukatsky [6,7].

It is known that the group $\operatorname{Dif} f(M)$ is topological group in compact open topology [18, p. 270], [13]. In this paper we investigate the groups $\operatorname{Diff}_{F}(M)$ of diffeomorphisms and the group $I s o_{F}(M)$ of isometries of foliated manifold $(M, F)$ with compact open topology. Also it is introduced new topology on $\operatorname{Dif} f_{F}(M)$ called $F$-compact open topology and we will show that some subgroups of Dif $f_{F}(M)$ are topological groups with $F$ - compact open topology.

\section{$\S 1$. Main part}

Let $M$ be a connected Riemannian $C^{\infty}$-manifold of dimension $n$.

Definition 1. A foliation $F$ on $\mathrm{M}$ of dimension $k$ (codimension $n-k$ ) is a partition of $M$ into arcwise connected subsets $L_{\alpha}$ with the following properties: 
(1) $M=\bigcup L_{\alpha}$;

(2) $L_{\alpha} \bigcap L_{\beta}=\emptyset$ if $\alpha \neq \beta$;

(3) for every point $p \in M$ there is an open neighborhood $U$ of $p$ and a chart $x=$ $=\left(x_{1}, x_{2}, \ldots, x_{k}, y_{1}, y_{2}, \ldots, y_{n-k}\right)$ such that for each leaf $L_{\alpha}$ the connected components of $L_{\alpha} \cap U$ are defined by the equations $y_{1}=$ const, $y_{2}=$ const, $\ldots, y_{n-k}=$ const.

Such a chart is a distinguished chart.

The connected components of the sets $y_{1}=$ const, $y_{2}=$ const, $\ldots, y_{n-k}=$ const in a distinguished chart are called plaques (plates) of $F$. Fixing $y_{1}=$ const, $y_{2}=$ const, $\ldots, y_{n-k}=$ const, the map $x \rightarrow(x, y))$ is a smooth embedding, therefore the plaques are connected $k$-dimensional submanifolds of $\mathrm{M}$. This shows that each leaf $L_{\alpha}$ is union of plaques and there exists differentiable structure $\sigma_{\alpha}$ on $L_{\alpha}$ such that $\left(L_{\alpha}, \sigma_{\alpha}\right)$ is a $k$ - dimensional connected manifold. Note that the canonical injection $i:\left(L_{\alpha}, \sigma_{\alpha}\right) \rightarrow M$ is an immersion, but it is not necessarily an embedding [20].

An example of a foliation is given by a smooth submersion $f: M \rightarrow B$, where $B-(n-k)$ dimensional manifold. The connected components of the inverse images of the points of $y \in B$ define a $k$-dimensional foliation of $M$.

We will denote by $(M, F)$ manifold $M$ with $k$ - dimensional foliation $F$ on $M$. Let $L(p)$ be a leaf of the foliation $F$ passing through point the $p, T_{p} F$ is the tangent space to the the leaf $L(p)$ at $p$.

We get subbundle (smooth distribution) $T F=\left\{T_{p} F: p \in M\right\}$ of the tangent bundle $T M$ of the manifold $M$.

Let us denote by $V(M), V(F)$ the set of smooth sections of bundles $T M, T F$ respectively. The set $V(F)$ consists of vector fields tangent to the foliation $F$. The set $V(M)$ is a Lie algebra with respect to the Lie bracket of vector fields, the set $V(F)$ is a Lie subalgebra of $V(M)$.

Definition 2. If for the some $C^{r}$ - diffeomorphism $\varphi: M \rightarrow M$ the image $\varphi\left(L_{\alpha}\right)$ of any leaf $L_{\alpha}$ of foliation $F$ is a leaf of foliation $F$, we say that the $\varphi$ is $C^{r}$ - diffeomorphism of foliated manifold and write as $\varphi:(M, F) \rightarrow(M, F)$.

Let's denote as $\operatorname{Dif} f_{F}(M)$ the set of all $C^{r}$ - diffeomorphisms of foliated manifold $(M, F)$, where $r \geq 0$. The group $\operatorname{Diff}_{F}(M)$ is subgroup of $\operatorname{Diff}(M)$ and therefore it is topological group in compact open topology.

Example 1. Let $M=\mathbb{R}^{2}(x, y)$ is a Euclidean plane with the Cartesian coordinates $(x, y)$. Leaves $L_{\alpha}$ of foliation $F$ are given by the equations $x^{2}-y=\alpha=$ const. Then the plan diffeomorphism $\varphi: \mathbb{R}^{2} \rightarrow \mathbb{R}^{2}$ determined by the formula

$$
\varphi(x, y)=(x, y+\lambda f(x, y))
$$

is diffeomorphism of foliated plane $\left(\mathbb{R}^{2}, F\right)$, for every $\lambda \in R$, such that $\lambda \neq 1$, where $f(x, y)=$ $=x^{2}-y$. It sends a leaf $L_{\alpha}$ to leaf $L_{(1-\lambda) \alpha}$. It is easy to check that in fact $\varphi: \mathbb{R}^{2} \rightarrow \mathbb{R}^{2}$ is an isometry of foliated plane $\left(\mathbb{R}^{2}, F\right)$.

Let $(M, F)$ be a foliated manifold, where $F$ is $k$-dimensional smooth foliation where $0<k<n$. Recall a vector field $X$ is called a foliated field if for every vector field $Y$, tangent to $F$, Lie brocket $[X, Y]$ also is tangent to $F$. It is known that flow of every foliated field consists of diffeomorphisms of foliated manifold $(M, F)$ [20]. The set $L(M, F)$ of foliated vector fields is a Lie subalgebra of Lie algebra $V(M)$ [8, p. 35]. It follows from here that the group $\operatorname{Dif} f_{F}(M)$ contains the Lie group for which the Lie algebra is an algebra $L(M, F)$. 
For foliated plane from example-1 vector field $X=\left(x^{2}-y\right) \frac{\partial}{\partial y}$ is foliated field and its flow consists of diffeomorphisms $\varphi^{t}:(x, y) \in \mathbb{R}^{2} \rightarrow\left(x, x^{2}-e^{-t}\left(x^{2}-y\right)\right) \in \mathbb{R}^{2}$ of foliated plane $\left(\mathbb{R}^{2}, F\right)$. Every diffeomorphism $\varphi^{t}:(x, y) \in \mathbb{R}^{2} \rightarrow\left(x, x^{2}-e^{-t}\left(x^{2}-y\right)\right) \in \mathbb{R}^{2}$ sends a leaf $L_{\alpha}$ to leaf $L_{e^{-t} \alpha}$.

As shown in the following simple example, from differentiability of mapping on each leaf does not follow it's differentiability on all manifold $M$.

Example 2. Let $M=\mathbb{R}^{2}(x, y)$ is a Euclidean plane with the Cartesian coordinates $(x, y)$. Leaves $L_{\alpha}$ of foliation $F$ are given by the equations $y=\alpha=$ const. Then the plan homeomorphism $\varphi: \mathbb{R}^{2} \rightarrow \mathbb{R}^{2}$ determined by the formula $\varphi(x, y)=\left(x+y, y^{\frac{1}{3}}\right)$ is a diffeomorphism on every leaf of the foliation $F$, but is not a diffeomorphism of plane.

Theorem 1. Let $(M, F)$ be a foliated manifold where $M$ is a smooth connected finitedimensional manifold. Then the group Dif $f_{F}(M)$ is a closed subgroup of Dif $f(M)$ in compact open topology.

P r o o f. Since a manifold $M$ is a separable metric space for $r \geq 0$ the set $C^{r}(M)$ of all $C^{r}$ maps of manifold $M$ onto itself with compact open topology has complete metric and a countable base [5, p. 35], [4, p. 202]. Consequently topological space $\operatorname{Dif} f(M)$ with induced compact open topology has a countable base. Thus we can use sequences to prove the theorem.

Let us assume that sequence $f_{i} \in \operatorname{Dif} f_{F}(M)$ convergences to $f \in \operatorname{Diff}(M)$ at $i \rightarrow \infty$ in compact-open topology and $K \subset L$ is a connected compact subset of some leaf $L$ of foliation of $F$.

We can assume that $f(K)$ is contained in a distinguished chart $(U, x, y)$. Then sets $f_{i}(K)$ are contained in local plaques which are determined by equations $y_{1}=c_{1}^{i}, y_{2}=c_{2}^{i}, \ldots, y_{n-k}=c_{n-k}^{i}$. It follows from here the set $f(K)$ is contained in the plaque which is determined by equations $y_{1}=$ const, $y_{2}=$ const, $\ldots, y_{n-k}=$ const. Hence the set $f(K)$ is subset of some leaf of foliation $F$.

In the case when $f(K)$ is not contained in a distinguished chart we can cover $f(K)$ with finite number of distinguished charts and by using the local coordinate system of a distinguished chart we get the set $f(K)$ is contained in some leaf of foliation $F$.

Now we consider case when $K \subset L$ is not connected compact, let $K_{1}$ and $K_{2}$ be two of its connected components. Assume the $f\left(K_{1}\right)$ is contained in a leaf $L_{1}$, the set $f\left(K_{2}\right)$ is contained in a leaf $L_{2}$. Since every leaf of foliation is arcwise connected by definition,there exists continuos path $\varphi:[0,1] \rightarrow L$ from $K_{1}$ to $K_{2}$, say $\varphi(0) \in K_{1}$ and $\varphi(1) \in K_{2}$. We cover compact $\varphi([0,1])$ with finite number of distinguished charts and by using the local coordinate system of a distinguished chart we get the set $\varphi([0,1])$ is contained in some leaf of foliation $F$. It follows from here that $L_{1}=L_{2}$, i. e., $f(K)$ is contained in some leaf of foliation $F$.

Every leaf of foliation as manifold is paracompact topologycal space and hence it can be written as $L=\bigcup_{1}^{\infty} K_{j}$, where $K_{j}$ - are compact subsets of $L$ and $K_{1} \subset K_{2} \subset \ldots$

We can repeat this process for compacts $K_{j}$ and get all the sets $f\left(K_{j}\right)$ are contained in the same leaf of foliation $F$. It follows the set $f(L)$ is a subset of some leaf $L^{\prime}$.

Since $L$ is $k$-dimensional manifold [19, p. 86], canonical injection $i:\left(L_{\alpha}, \sigma_{\alpha}\right) \rightarrow M$ is an immersion and $f$ is a homeomorphism, the image $f(L)$ is a $k$-dimensional topological manifold, i. e., every point of $f(L)$ has a neighborhood in $f(L)$ homeomorphic to an open ball in $R^{k}$. Hence the set $f(L)$ is a open subset of $L^{\prime}$.

We will show it is closed subset of $L^{\prime}$. Let sequence $q_{i} \in f(L)$ convergences to a point $q$ in topology of $L^{\prime}, U_{q}$ be a neighborhood of the point of $q$ with compact closure in $L^{\prime}$. As Diff $(M)$ is topological group in compact open topology, it follows from $f_{i} \rightarrow f$ at $i \rightarrow \infty$ that $f_{i}^{-1} \rightarrow f^{-1}$ 
at $i \rightarrow \infty$. By using arguments above we get $f^{-1}\left(U_{q}\right)$ is contained in the some leaf. But the set $U_{q}$ contains points $q_{i}$ for large $i$. It follows from here that the set $f^{-1}\left(U_{q}\right)$ is contained in the leaf $L$. Then sequence $p_{i}=f^{-1}\left(q_{i}\right) \in L$ convergences to a point $p=f^{-1}(q)$ in topology of $L$. Therefore $q=f(p) \in f(L)$. As $L^{\prime}$ is connected manifold we have $f(L)=L^{\prime}$. From here we have $f \in \operatorname{Diff}_{F}(M)$.

The closedness of the set Dif $f_{F}(M)$ allows us to state the following corollary [2, p. 30].

Corollary 1. Factor space Dif $(M) / D$ if $f_{F}(M)$ is regular homogeneous topological space.

Let $M$ be a smooth connected finite-dimensional Riemannian manifold.

Definition 3. An isometry $\varphi: M \rightarrow M$ is called an isometry of foliated manifold $(M, F)$ if it is diffeomorphism of foliated manifold $(M, F)$.

We will denote by $I_{s o_{F}}(M)$ the set of all $C^{r}$-isometries of foliated manifold $(M, F)$, where $r \geq 0$. We have that $\operatorname{Iso}_{F}(M)=\operatorname{Diff}_{F}(M) \bigcap I s o(M)$.

Example 3. Let $M=\mathbb{R}^{2}(x, y)$ is a Euclidean plane with the Cartesian coordinates $(x, y)$, foliation $F$ are given by the equations $x^{2}-y=\alpha=$ const. Then the plan isometry $\varphi: \mathbb{R}^{2} \rightarrow \mathbb{R}^{2}$ determined by the formula

$$
\varphi_{\lambda}(x, y)=(x, y+\lambda)
$$

is isometry of foliated plane $\left(\mathbb{R}^{2}, F\right)$ for every $\lambda \in \mathbb{R}$. It sends a leaf $L_{\alpha}$ to $L_{\alpha-\lambda}$. Family of isometries $\varphi_{\lambda}$ is the flow of Killing vector field $X=\frac{\partial}{\partial y}$.

Let us recall that vector field $X$ on riemannian manifold $(M, g)$ is called Killing field if its flow consists of isometries of Riemannian manifold $(M, g)$, that is $L_{X} g=0$, where $g$ is riemannian metric, $L_{X} g$ denotes Lie derivative of the metric $g$ with respect to $X$. Geometry of orbits of Killing vector fields is studied in $[10-12,14]$. If $X$ is foliated Killing vector field, it's flow consists of isometries of foliated manifold $(M, F)$.) The set $K(M, F)$ of foliated Killing vector fields is a Lie subalgebra of Lie algebra $L(M, F)$. It follows from here that the group $I s_{F}(M)$ contains the Lie group for which the Lie algebra is an algebra $K(M, F)$.

It is known that limit of isometries is an isometry. By using this fact as direct consequence of Theorem 1 and the definition of the induced topology we have following theorem.

Theorem 2. Let $(M, F)$ be a foliated manifold where $M$ is a smooth connected finitedimensional Riemannian manifold. Then the group $I_{s o}(M)$ is closed subset of Iso $(M)$ in compact open topology.

From the Theorem 2 and Cartan theorem [17, p. 242] it follows very important fact that the group $\operatorname{Iso}_{F}(M)$ is Lie group. Really Cartan's theorem states that on a closed subgroup of a Lie group there exists a differential structure with respect to which the closed subgroup is a Lie subgroup of a given Lie group. This fact we formulate as theorem in following form.

Theorem 3. Let $(M, F)$ be a foliated manifold where $M$ is a smooth connected finitedimensional Riemannian manifold. Then the group $\operatorname{Iso}_{F}(M)$ is Lie subgroup of Lie group Iso $(M)$.

We will introduce some topology on the group Dif $f_{F}(M)$, which depends on foliation $F$ and coincides with compact open topology when $F$ is $n$-dimensional foliation.

Let $\left\{K_{\lambda}\right\}$ be a family of all compact sets where each $K_{\lambda}$ is a subset of some leaf $L_{\lambda}$ of foliation $F$ and let $\left\{U_{\beta}\right\}$ is the family of all open sets on $M$. We consider for each pair $K_{\lambda}$ 
and $U_{\beta}$ set of all mappings $f \in \operatorname{Dif} f_{F}(M)$ for which $f\left(K_{\lambda}\right) \subset U_{\beta}$. This set of mappings we denote through $\left[K_{\lambda}, U_{\beta}\right]=\left\{f: M \rightarrow M \mid f\left(K_{\lambda}\right) \subset U_{\beta}\right\}$.

It isn't difficult to show that every possible finite intersections of sets of the form $\left[K_{\lambda}, U_{\beta}\right]$ forms a base for some topology. This topology we call foliated compact open topology or in brief $F$-compact open topology. The space Dif $f_{F}(M)$ with $F$-compact open topology is Hausdorff topologycal space [13]. Since $K$ runs only over all compact subsets of leaves, $F$-compact open topology on $\operatorname{Dif} f_{F}(M)$ is weaker than induced from $\operatorname{Dif} f(M)$ usual compact open topology.

Theorem 4. Let $(M, F)$ be a foliated manifold, where $M$ is a smooth connected finite-dimensional Riemannian manifold. Then the group $\operatorname{Iso}_{F}(M)$ is a topological group with F-compact open topology.

P r o o f. We denote through $d(x, y)$ the distance between points $x$ and $y$, determined by Riemannian metric $g$.

First of all we will show that mapping $(g, h) \rightarrow g \circ h$ is continuous mapping for $g, h \in$ $\in \operatorname{Iso}_{F}(M)$.

Let $g \circ h \in[K, G]$, where $K$ is a compact on some leaf of foliation $F, G$ is a open subset of $M$. Since $K^{\prime}=g(h(K))$ is a compact set, there is $\varepsilon>0$ such that neighborhood $V_{\varepsilon}=$ $\left.=\left\{y \in M: d\left(y, K^{\prime}\right)\right)<\varepsilon\right\}$ of $K^{\prime}$ is contained in $G$, where $d\left(y, K^{\prime}\right)=\inf \left\{d(y, z): z \in K^{\prime}\right\}$.

Now we put

$$
U_{\varepsilon_{1}}(h)=\left\{h^{\prime} \in I s o_{F}^{r}(M): d\left(h^{\prime}(x), h(x)\right)<\varepsilon_{1}, \quad \forall x \in K\right\}
$$

and

$$
U_{\varepsilon_{2}}(g)=\left\{g^{\prime} \in I s o_{F}^{r}(M): d\left(g^{\prime}(y), g(y)\right)<\varepsilon_{2}, \quad \forall y \in h(K)\right\}
$$

where $\varepsilon_{1}>0, \varepsilon_{2}>0$, and $\varepsilon_{1}+\varepsilon_{2}<\varepsilon$.

The set $U_{\varepsilon_{1}}(h)$ is a neighborhood of $h$ in $F$-compact open topology as $U_{\varepsilon_{1}}(h)=\left[K, V_{\varepsilon_{1}}\right]$, the set $U_{\varepsilon_{2}}(g)$ is a neighborhood of $g$ in $F$-compact-open topology, as $U_{\varepsilon_{2}}(h)=\left[h(K), V_{\varepsilon_{2}}^{\prime}\right]$, where $V_{\varepsilon_{1}}=\left\{x \in M: d(x, h(K))<\varepsilon_{1}\right\}, V_{\varepsilon_{2}}^{\prime}=\left\{y \in M: d\left(y, K^{\prime}\right)<\varepsilon_{2}\right\}$.

If $h^{\prime} \in U_{\varepsilon_{1}}(h), g^{\prime} \in U_{\varepsilon_{2}}(g), x \in K, y=h(x)$ and $y^{\prime}=h^{\prime}(x)$ then

$$
d\left(g^{\prime}\left(h^{\prime}(x)\right), g(h(x))\right)=d\left(g^{\prime}\left(y^{\prime}\right), g(y)\right) \leq d\left(g^{\prime}\left(y^{\prime}\right), g^{\prime}(y)\right)+d\left(g^{\prime}(y), g(y)\right) .
$$

Since $g, g^{\prime}$ are isometries we have

$$
d\left(g^{\prime}\left(y^{\prime}\right), g^{\prime}(y)\right)+d\left(g^{\prime}(y), g(y)\right) \leq d\left(y^{\prime}, y\right)+\varepsilon_{2} \leq \varepsilon_{1}+\varepsilon_{2}<\varepsilon .
$$

It follows from here that if $\left(g^{\prime}, h^{\prime}\right) \in U_{\varepsilon_{2}}(g) \times U_{\varepsilon_{1}}(h)$, then $g^{\prime} \circ h^{\prime} \in[K, G]$.

Now we shall prove, that mapping $\chi: f \rightarrow f^{-1}$ is continuous.

For this purpose we shall prove, that the full inverse image $\chi^{-1}(A)$ of the open set $A \subset \operatorname{Iso}_{F}(M)$ is a open set. Really it is enough to show this fact when $A$ is an element of prebase, i. e., $A=\left\{f \in I s_{F}(M): f(K) \subset V\right\}$ where $K$ is compact subset of the leaf $L$ of foliation $F, V$ is open set. In this case $\chi^{-1}(A)=\left\{f \in I s_{F}(M): f^{-1}(K) \subset V\right\}$.

We shall show, that $\chi^{-1}(A)$ is a open set in $F$-compact open topology.

Let $g \in \chi^{-1}(A), U-$ a neighborhood of $g^{-1}(K)$ in $M$ with compact closure such, that $\bar{U} \subset V$. We put $K_{1}=g^{-1}(K), U_{g}=\left\{h \in I s o_{F}^{r}(M): d(g(x), h(x))<\frac{\varepsilon}{2}, \quad \forall x \in K_{1}\right\}$, where $\varepsilon=\operatorname{dist}\left(K_{1}, M \backslash U\right)=\inf \left\{d(x, y): x \in K_{1}, y \in(M \backslash U)\right\}$.

We need to show that if $h \in U_{g}$ then $h^{-1}(K) \subset U$. In other words, we must show that $h^{-1}(y) \in U$ for all $y \in K$. Indeed, since $g^{-1}(y) \in K_{1}$,

$$
d\left(y, h\left(g^{-1}(y)\right)=d\left(g\left(g^{-1}(y)\right), h\left(g^{-1}(y)\right)\right)<\frac{\varepsilon}{2} .\right.
$$


Since $\mathrm{h}$ is an isometry we have $d\left(y, h\left(g^{-1}(y)\right)\right)=d\left(h^{-1}(y), h^{-1} \cdot h \cdot g^{-1}(y)\right)=d\left(h^{-1}(y), g^{-1}(y)\right)$. Thus $d\left(h^{-1}(y), g^{-1}(y)\right)<\frac{\varepsilon}{2}$ and $h^{-1}(y)$ belongs to a $\frac{\varepsilon}{2}$-neighborhood of $g^{-1}(y)$ and therefore of $\frac{\varepsilon}{2}$-neighborhood of $K_{1}$. Hence it is contained in $U$, that is $h^{-1}(y) \in U$.

Let's denote as $\operatorname{Dif} f_{F}^{0}(M)$ set of all $C^{r}$ diffeomorphisms $g \in D$ if $f_{F}(M)$ of foliated manifold $(M, F)$, such that $g\left(L_{\alpha}\right)=L_{\alpha}$ for every $L_{\alpha}$ leaf of foliation $F$. Flow of every tangent vector field consists of diffeomorphisms of foliated manifold $(M, F)$, which belong to the group Dif $f_{F}^{0}(M)$. It follows from here that the group $\operatorname{Dif} f_{F}^{0}(M)$ contains the Lie group for which the Lie algebra is an algebra $V(F)$.

It can be proven following theorem.

Theorem 5. Let $(M, F)$ be a foliated manifold, where $M$ is a smooth, connected and finite-dimensional manifold. Assume every leaf $L_{\alpha}$ of $F$ is a proper, i. e., canonic injection $i: L_{\alpha} \rightarrow M$ is an embedding. Then the group Dif $f_{F}^{0}(M)$ is a topological group with F-compact open topology.

P r o o f. First of all we will show that mapping $(g, h) \rightarrow g \circ h$ is continuous mapping for $g, h \in \operatorname{Diff}_{F}^{0}(M)$.

Let $g \circ h \in[K, G]$, where $K$ is a compact subset of some leaf $L$ of foliation $F, G$ is a open subset of $M$. Then $h(K) \subset g^{-1}(G)$ and since $h(K)$ is a compact set, there is open neighborhood $A$ of $h(K)$ in the leaf $h(L)$ with compact closure, i. e., $\bar{A}$ compact in $h(L)$. Since the leaf $h(L)$ is a proper there exists open subset $A^{\prime}$ of $M$ such that $A=h(L) \cap A^{\prime}$. Since $h$ is leaf-preserving map we have $A=L \cap A^{\prime}$. If $h^{\prime} \in\left[K, A^{\prime}\right]$, then $h^{\prime}(K) \subset A^{\prime} \cap h(L)$ and $h(K) \subset A$. It follows from here that if $\left(g^{\prime}, h^{\prime}\right) \in[\bar{A}, G] \times\left[K, A^{\prime}\right]$, then $g^{\prime} \circ h^{\prime} \in[K, G]$. Thus, the inverse image of the open set under the mapping $(g, h) \rightarrow g \circ h$ is open; therefore, the map $(g, h) \rightarrow g \circ h$ is continuous.

We shall prove, that mapping $\chi: f \rightarrow f^{-1}$ is continuous.

Let $g$ be complete Riemannian metric on $M$. It is known, smooth manifold $M$ possess complete Riemannian metric [3, p. 186], [4].

The restriction of the Riemannian metric $g$ to $T_{p} F$ for all $p \in M$ induces complete Riemannian metric on the leaves [11].

Let $L_{\alpha}$ be a some leaf of foliation $F, d_{\alpha}$ is the distance on $L_{\alpha}$, determined by induced Riemannian metric $g_{\alpha}$.

Now using complete Riemannian metric $g_{\alpha}$ we shall prove, that mapping $\chi: f \rightarrow f^{-1}$ is continuous. For this purpose we shall prove, that the full inverse image $\chi^{-1}(A)$ of the open set $A \subset \operatorname{Diff}_{F}^{0}(M)$ is a open set.

Really it is enough to show this fact when $A$ is an element of prebase, i. e., $A=$ $=\left\{f \in \operatorname{Dif} f_{F}^{0}(M): f(K) \subset V\right\}$ where $K$ is compact subset of a leaf $L_{\alpha}, V$ is open subset of $M$. In this case $\chi^{-1}(A)=\left\{f \in D i f f_{F}^{0}(M): f^{-1}(K) \subset V\right\}$.

We shall show, that $\chi^{-1}(A)$ is a open set in $F$-compact open topology.

Let $g \in \chi^{-1}(A), U$ a neighborhood of $g^{-1}(K)$ in $L_{\alpha}$ with compact closure $\bar{U}$ in topology of $L_{\alpha}$ such, that $\bar{U} \subset V$.

We put $U(g)=\left\{g \in D i f f_{F}^{0}(M): d_{\alpha}(g(x), h(x))<\frac{\varepsilon}{2}, \forall x \in \bar{U}\right\}$, where

$$
\varepsilon=d_{\alpha}\left(K, L_{\alpha} \backslash g(U)\right)=\inf \left\{d_{\alpha}(x, y): x \in K, y \in\left(L_{\alpha} \backslash g(U)\right)\right\} .
$$

Let's show, that if $h \in U(g)$ takes place $h^{-1}(K) \subset V$, i. e., $U(g) \subset \chi^{-1}(A)$. We shall show, that $h^{-1}(K) \subset U$.

Let's assume, it is not true. Let for the some $h \in U(g)$ there exists a point $y \in K$ such that $h^{-1}(y) \in L_{\alpha} \backslash U$, i.e., $y \in L_{\alpha} \backslash h(U)$. Then by virtue of that $g^{-1}(y) \in U$, takes place $d_{\alpha}\left(y=g\left(g^{-1}(y)\right), h\left(g^{-1}(y)\right)\right)<\frac{\varepsilon}{2}$.

Let $\gamma$ - the shortest geodesic in $L_{\alpha}$ (by virtue of completeness $\left(L_{\alpha}, g_{\alpha}\right)$ ) there exists a shortest geodesic between any two points) going from the point $y$ to the point $h\left(g^{-1}(y)\right)$, and 
$z \in \gamma \cap \partial(h(U))$. Then $h^{-1}(z) \in \bar{U}$ and besides $\left.d_{\alpha}\left(g\left(h^{-1}(z)\right), h\left(h^{-1}(z)\right)\right)\right)<\frac{\varepsilon}{2}$. In addition $d_{\alpha}(y, z)<\frac{\varepsilon}{2}$. Hence $d_{\alpha}\left(y, g\left(h^{-1}(z)\right)\right) \leq d_{\alpha}(y, z)+d_{\alpha}\left(z, g\left(h^{-1}(z)\right)\right)<\varepsilon$.

But on the other hand, by virtue of that $z \notin h(U)$, we have $g\left(h^{-1}(z)\right) \in M \backslash g(U)$. Since $y \in K \subset g(U), g\left(h^{-1}(z)\right) \notin g(U)$ and $\varepsilon=d_{\alpha}\left(K, L_{\alpha} \backslash g(U)\right)$ we have $d_{\alpha}\left(y, g\left(h^{-1}(z)\right)\right) \geq \varepsilon$. This contradiction shows that $h^{-1}(K) \subset U$. Hence, $U(g) \subset \chi^{-1}(A)$.

Proposition 1. Let $(M, F)$ be a foliated manifold, where $M$ is a smooth, connected and finitedimensional manifold. Assume all leaves of foliated manifold $(M, F)$ are closed subsets of $M$. Then the group Dif $f_{F}^{0}(M)$ is closed subset of Dif $f_{F}(M)$ in F-compact open topology.

P r o o f. Let us consider the set $A=D i f f_{F}(M) \backslash D i f f_{F}^{0}(M)$ and show that the set $A$ is open subset of Dif $f_{F}(M)$ in $F$-compact open topology. If $f \in A$ then there exists a leaf $L$ of foliation $F$ such that $f(L)=L^{\prime}$, and $L \neq L^{\prime}, L^{\prime}-$ another leaf of foliation $F$.

Let $K$ be a compact subset of the leaf $L, \varepsilon_{i}$ - real numbers, $i=1,2, \ldots$, and $\varepsilon_{i} \rightarrow 0$ at $i \rightarrow \infty$.

We put

$$
U_{i}(f)=\left\{g \in \operatorname{Diff}_{F}(M): d(g(x), f(x))<\varepsilon_{i}, \forall x \in K\right\}, \quad V_{\varepsilon_{i}}=\left\{x \in M: d(x, f(K))<\varepsilon_{i}\right\},
$$

where $d$ - the distance on $M$, determined by Riemannian metric $g$. The set $U_{i}(f)$ is a open subset of Dif $f_{F}(M)$ in $F$-compact open topology since $U_{i}(f)=\left[K, V_{\varepsilon_{i}}\right]$.

If for some $i$ the set $U_{i}(f)$ does not contain elements of $\operatorname{Dif} f_{F}^{0}(M)$ then the set $U_{i}(f) \subset A$ and $f$ is a interior point of $A$.

Assume that for all $i$ the set $U_{i}(f)$ contains element $f_{i}$ of $\operatorname{Dif} f_{F}^{0}(M)$. Then sequence $f_{i}(x)$ convergences to $f(x)$ at $i \rightarrow \infty$ for every $x \in K$. It follows from here $f(x) \in \bar{L}$, where $\bar{L}-$ closure of $L$ in $M$. Since $\bar{L}=L$, we have $f(x) \in L$. On the other hand since $f(L)=L^{\prime}$ and $L \cap L^{\prime}=\emptyset$ it follows from here $f(K) \subset L^{\prime}$. This contradiction shows that for large $i$ the set $U_{i}(f)$ does not contain elements of $\operatorname{Dif} f_{F}^{0}(M)$ and $U_{i}(f) \subset A$, i. e., element $f$ is a interior point of $A$.

\section{REFERENCES}

1. Arnold V. Sur la géométrie différentielle des groupes de Lie de dimension infinite et ses applications à l'hidrodynamique des fluides parfaits, Annales de l'Institut Fourier, 1966, vol. 16, pp. 318-361. https://doi.org/10.5802/aif.233

2. Chevalley C. Theory of Lie groups. I., Princeton: Princeton University press, 1966. https://archive.org/details/in.ernet.dli.2015.86469/page/n7/mode/2up

3. Gromoll D., Klingenberg W., Meyer W. Riemannsche Geometrie im Großen, Berlin: Springer, 1968. https://doi.org/10.1007/978-3-540-35901-2

4. Helgason S. Differential geometry, Lie groups and symmetric spaces, Toronto: Academic Press, 1978.

5. Hirsch M. W. Differential topology, New York: Springer, 1976.

6. Lukatskii A. M. Finite generation of groups of diffeomorphisms, Russian Mathematical Surveys, 1978, vol. 33, no. 1, pp. 207-261. https://doi.org/10.1070/RM1978v033n01ABEH002248

7. Lukatsky A.M. Investigation of the geodesic flow on an infinite-dimensional Lie group by means of the coadjoint action operator, Proceedings of the Steklov Institute of Mathematics, 2009, vol. 267, pp. 195-204. https://doi.org/10.1134/s0081543809040166

8. Molino P. Riemannian foliations, Boston-Basel: Burkhauser, 1988.

9. Myers S. B., Steenrod N. E. The group of isometries of a Riemannian manifold, Annals of Mathematics. Second Series, 1939, vol. 40, no. 2, pp. 400-416. https://doi.org/10.2307/1968928

10. Narmanov A. Ya., Saitova S. S. On geometry of vector fields, Journal of Mathematical Sciences, 2020, vol. 245, pp. 375-381. https://doi.org/10.1007/s10958-020-04699-z 
11. Narmanov A. Y. Geometry of orbits of vector fields and singular foliations, Contemporary Mathematics. Fundamental Directions, 2019, vol. 65, issue 1, pp. 54-71 (in Russian). https://doi.org/10.22363/2413-3639-2019-65-1-54-71

12. Narmanov A., Rajabov E. On the geometry of orbits of conformal vector fields, Journal of Geometry and Symmetry in Physics, 2019, vol. 51, pp. 29-39. https://doi.org/10.7546/jgsp-51-2019-29-39

13. Narmanov A., Sharipov A. On the group of foliation isometries, Methods of Functional Analysis and Topology, 2009, vol. 15, pp. 195-209. http://mfat.imath. kiev.ua/article/?id=435

14. Narmanov A. Ya., Saitova S. S. On the geometry of orbits of Killing vector fields, Differential Equations, 2014, vol. 50, pp. 1584-1591. https://doi.org/10.1134/s0012266114120027

15. Omori H. On the group of diffeomorphisms on a compact manifold, Proc. Symp. Pure Math., 1970, vol. 15, pp. 167-183. https: //mathscinet.ams.org/mathscinet-getitem?mr=0271983

16. Omori H. Group of diffeomorphisms and thier subgroups, Trans. Amer. Math. Soc., 1973, vol. 179, pp. 85-122. https://doi.org/10.1090/s0002-9947-1973-0377975-0

17. Postnikov M. M. Lectures on geometry. Semester V. Lie groups and Lie algebras, Moscow: Nauka, 1982. https://archive.org/details/postnikovliegroups/page/n5/mode/2up

18. Rokhlin V. A., Fuks D. B. Initial course of topology. Geometrical chapters, Moscow: Mir, 1977.

19. Tamura I. Topology of foliations: An introduction, Providence, Rhode Island: American Mathematical Society, 1992.

20. Tondeur Ph. Foliations on Riemannian manifolds, New York: Springer, 1988.

Received 01.02 .2020

Narmanov Abdigappar Yakubovich, Doctor of Physics and Mathematics, Professor, Department of Geometry, National University of Uzbekistan, ul. Universitetskaya, 4, Tashkent, 100174, Uzbekistan.

E-mail: narmanov@yandex.ru

Zoyidov Azam Nurilloevich, Assistant Lecturer, Department of Geometry, National University of Uzbekistan, ul. Universitetskaya, 4, Tashkent, 100174, Uzbekistan.

E-mail: zoyid.azam.math@gmail.com

Citation: A. Y. Narmanov, A. N. Zoyidov. On the group of diffeomorphisms of foliated manifolds, Vestnik Udmurtskogo Universiteta. Matematika. Mekhanika. Komp'yuternye Nauki, 2020, vol. 30, issue 1, pp. 49-58. 


\section{А. Я. Нарманов, А. Н. Зоидов}

\section{О группе диффеоморфизмов слоеных многообразий}

Ключевые слова: многообразие, слоение, группа диффеоморфизмов, компактно открытая топология.

УДК 517.977

DOI: $10.35634 / \mathrm{vm} 200104$

В настоящее время теория слоений является интенсивно развивающимся разделом современной дифференциальной геометрии, что показывают многочисленные исследования по теории слоений. Целью нашей работы является изучение структуры группы диффеоморфизмов $\operatorname{Dif} f_{F}(M)$ и группы изометрий $I s o_{F}(M)$ слоеного многообразия $(M, F)$. Показано, что группа $D i f f_{F}(M)$ является замкнутой подгруппой группы $\operatorname{Diff}(M)$ диффеоморфизмов многообразия $M$ в компактно-открытой топологии, а также доказана,что группа изометрий $I s o_{F}(M)$ слоеного многообразия является группой Ли. Введена новая топология на $\operatorname{Diff} F(M)$, которая зависит от слоения $F$ и называется $F$ компактно открытой топологией. Доказано, что некоторые подгруппы группы $\operatorname{Dif} f_{F}(M)$ являются топологическими группами с $F$-компактно открытой топологией.

\section{СПИСОК ЛИТЕРАТУРЫ}

1. Arnold V. Sur la géométrie différentielle des groupes de Lie de dimension infinite et ses applications à l'hidrodynamique des fluides parfaits // Annales de l'Institut Fourier. 1966. Vol. 16. P. 318-361. https://doi.org/10.5802/aif.233

2. Chevalley C. Theory of Lie groups. I. Princeton: Princeton University press, 1966. https://archive.org/details/in.ernet.dli.2015.86469/page/n7/mode/2up

3. Gromoll D., Klingenberg W., Meyer W. Riemannsche Geometrie im Großen. Berlin: Springer, 1968. https://doi.org/10.1007/978-3-540-35901-2

4. Helgason S. Differential geometry, Lie groups and symmetric spaces. Toronto: Academic Press, 1978.

5. Hirsch M. W. Differential topology, New York: Springer, 1976.

6. Lukatskii A.M. Finite generation of groups of diffeomorphisms // Russian Mathematical Surveys. 1978. Vol. 33. No. 1. P. 207-261. https://doi.org/10.1070/RM1978v033n01ABEH002248

7. Lukatsky A. M. Investigation of the geodesic flow on an infinite-dimensional Lie group by means of the coadjoint action operator // Proceedings of the Steklov Institute of Mathematics. 2009. Vol. 267. P. 195-204. https://doi.org/10.1134/S0081543809040166

8. Molino P. Riemannian foliations. Boston-Basel: Burkhauser, 1988.

9. Myers S. B., Steenrod N. E. The group of isometries of a Riemannian manifold // Annals of Mathematics. Second Series. 1939. Vol. 40. No. 2. P. 400-416. https://doi.org/10.2307/1968928

10. Narmanov A. Ya., Saitova S. S. On geometry of vector fields // Journal of Mathematical Sciences. 2020. Vol. 245. P. 375-381. https://doi.org/10.1007/s10958-020-04699-z

11. Narmanov A. Y. Geometry of orbits of vector fields and singular foliations // Contemporary Mathematics. Fundamental Directions. 2019. Vol. 65. Issue 1. P. 54-71 (in Russian). https: //doi.org/10.22363/2413-3639-2019-65-1-54-71

12. Narmanov A., Rajabov E. On the geometry of orbits of conformal vector fields // Journal of Geometry and Symmetry in Physics. 2019. Vol. 51. P. 29-39. https://doi.org/10.7546/jgsp-51-2019-29-39

13. Narmanov A., Sharipov A. On the group of foliation isometries // Methods of Functional Analysis and Topology. 2009. Vol. 15. P. 195-209. http://mfat.imath. kiev.ua/article/?id=435

14. Narmanov A. Ya., Saitova S. S. On the geometry of orbits of Killing vector fields // Differential Equations. 2014. Vol. 50. P. 1584-1591. https://doi.org/10.1134/S0012266114120027 
15. Omori H. On the group of diffeomorphisms on a compact manifold // Proc. Symp. Pure Math. 1970. Vol. 15. P. 167-183.

https: //mathscinet.ams.org/mathscinet-getitem?mr=0271983

16. Omori H. Group of diffeomorphisms and thier subgroups // Trans. Amer. Math. Soc. 1973. Vol. 179. P. 85-122. https://doi.org/10.1090/S0002-9947-1973-0377975-0

17. Postnikov M. M. Lectures on geometry. Semester V. Lie groups and Lie algebras. Moscow: Nauka, 1982. https://archive.org/details/postnikovliegroups/page/n5/mode/2up

18. Рохлин В. А., Фукс Д. Б. Начальный курс топологии: Геометрические главы. М.: Наука, 1977.

19. Tamura I. Topology of foliations: An introduction. Providence, Rhode Island: American Mathematical Society, 1992.

20. Tondeur Ph. Foliations on Riemannian manifolds. New York: Springer, 1988.

Поступила в редакцию 01.02 .2020

Нарманов Абдигаппар Якубович, д. ф.-м. н., профессор, кафедра геометрии, Национальный университет Узбекистана, 100174, Узбекистан, г. Ташкент, ул. Университетская, 4.

E-mail: narmanov@yandex.ru

Зоидов Азам Нуриллоевич, ассистент, кафедра геометрии, Национальный университет Узбекистана, 100174, Узбекистан, г. Ташкент, ул. Университетская, 4.

E-mail: zoyid.azam.math@gmail.com

Цитирование: А.Я. Нарманов, А.Н. Зоидов. О группе диффеоморфизмов слоеных многообразий // Вестник Удмуртского университета. Математика. Механика. Компьютерные науки. 2020. Т. 30. Вып. 1. С. 49-58. 